\title{
Adaptación del cuestionario de personalidad resistente en población mexicana
}

Adaptation of hardy personality questionnaire in mexican population

\author{
Adaptação do questionário de personalidade resistente \\ na população mexicana
}

Rosa M. Meda-Lara ${ }^{1}$

Carmen Yeo-Ayala ${ }^{2}$

Pedro Juárez-Rodríguez ${ }^{3}$

Andrés Palomera-Cháve ${ }^{4}$

Bernardo Moreno-Jiméne $z^{5}$

Recibido: 17 de agosto de 2020

Aprobado: 7 de diciembre de 2020

Publicado: 4 de julio de 2021

Cómo citar este artículo:

Meda-Lara, R.M., Yeo-Ayala, C., Juárez-Rodríguez, P., Palomera-Chávez, A y MorenoJiménez, B. (2021). Adaptación del cuestionario de personalidad resistente en población mexicana. Pensando Psicología, 17(2), 1-24. doi: https://doi.org/10.16925/2382-3984.2021.02.03

Artículo de investigación. https://doi.org/10.16925/2382-3984.2021.02.03

1 Departamento de Psicología Básica, Centro Universitario de Ciencias de la Salud. Universidad de Guadalajara. Jalisco, México.

Correo electrónico: rosa.meda@academicos.udg.mx

ORCID: https://orcid.org/0000-0001-8967-7138

Sierra Mojada 950, Col. Independencia, C.P. 44340, Guadalajara, Jalisco, México.

2 Departamento de Psicología Básica, Centro Universitario de Ciencias de la Salud. Universidad de Guadalajara. Jalisco, México.

ORCID: https://orcid.org/0000-0002-9567-9807

3 Departamento de Biología Molecular y Genómica, Centro Universitario de Ciencias de la Salud. Universidad de Guadalajara. Jalisco, México.

ORCID: https://orcid.org/0000-0001-5663-684X

4 Departamento de Psicología Básica, Centro Universitario de Ciencias de la Salud. Universidad de Guadalajara. Jalisco, México.

ORCID: https://orcid.org/0000-0003-0124-523X

5 Departamento Psicología Biológica y de la Salud, Facultad de Psicología. Universidad Autónoma de Madrid. Madrid, España.

ORCID: https://orcid.org/0000-0001-5360-0518 


\title{
Resumen
}

Introducción: El concepto de personalidad resistente tal como lo propone Kobasa queda definido por tres dimensiones, a saber: compromiso, control y reto. El cuestionario de personalidad resistente (CPR) originalmente fue diseñado para evaluar la personalidad resistente en el ámbito laboral. El CPR se ha adaptado y validado en diferentes países latinoamericanos; sin embargo, en México no se validado psicométricamente.

Objetivo: analizar las propiedades psicométricas de la adaptación del CPR en adultos mexicanos.

Metodología: se contó con una muestra no aleatoria de 357 adultos que reportaron estar sanos. Se adaptó el CPR a la forma de enfrentar la vida cotidiana.

Resultados: en el análisis factorial confirmatorio se estimaron tres modelos, de ellos el que mostró mejores índices de ajuste fue el trifactorial (control, implicación y reto) con un factor de segundo orden (personalidad resistente), la consistencia interna del CPR fue aceptable y la validez de constructo indicó correlaciones positivas de las dimensiones del CPR con las variables positivas de satisfacción con la vida, optimismo y autoeficacia general y correlaciones negativas con las variables patogénicas ansiedad y disfunción social.

Conclusiones: los resultados encontrados indican que el CPR tiene características psicométricas satisfactorias y que puede utilizarse para medir personalidad resistente (reto, control e implicación) en adultos mexicanos.

Palabras clave: personalidad resistente, adaptación, validación psicométrica, población mexicana.

\begin{abstract}
Introduction Hardy personality construct as proposed by Kobasa is defined by three dimensions: engagement, control, and challenge. It was Originally designed to assess Hardy personality (HPA) in work environment. HPA has been accepted and validated in different Latin American countries, nonetheless, in Mexico, its psychometric properties haven't been validated.
\end{abstract}

Aim: To analyze psychometric properties of HPA in Mexican adults.

Methodology: A non-randomized sample of 357 adults participated and reported being healthy. HPA was adapted to face ordinary life.

Results: Confirmatory factor analysis allowed to estimate three models, the one that showed better adjustment of fit was the trifactorial (engagement, control, and challenge) with a second-order factor (hardy personality), internal consistency of HPA was acceptable and construct validity indicated positive correlations in HPA dimensions with life satisfaction, optimism, and general efficacy, and negative correlations with axiety and social disfunction.

Conclusions: Results indicated that HPA has satisfactory psychometric properties and that can be used to assess hardy personality (engagement, control, and challenge) in Mexican adults.

Keywords: hardy personality, adaptation, psychometric validation, Mexican population.

\section{Resumo}

Introdução: 0 conceito de personalidade resistente proposto por Kobasa é definido por três dimensões, a saber: comprometimento, controle e desafio. O questionário de personalidade resistente (QPR) foi originalmente elaborado para avaliar a personalidade resistente no local de trabalho. O QPR foi adaptado e validado em diferentes países da América Latina; entretanto, no México, não foi validado psicometricamente.

Objetivo: Analisar as propriedades psicométricas da adaptação da RCP em adultos mexicanos.

Metodologia: Contou-se com uma amostra não aleatória de 357 adultos que relataram ser saudáveis. O QPR foi adaptado ao modo de enfrentar a vida cotidiana. 
Rosa M. Meda-Lara, Carmen Yeo-Ayala, Pedro Juárez-Rodríguez, Andrés Palomera-Chávez, 3 Bernardo Moreno-Jiménez

Resultados: na análise fatorial confirmatória foram utilizados três modelos, dos quais o que apresentou os melhores índices de ajuste foi o trifatorial (controle, envolvimento e desafio) com um fator de segunda ordem (personalidade resistente). A consistência interna do QPR foi aceitável e a validade de construto indicou correlações positivas das dimensões da QPR com as variáveis positivas de satisfação com a vida, otimismo e autoeficácia geral e correlações negativas com as variáveis patogênicas ansiedade e disfunção social.

Conclusões: os resultados encontrados indicam que o QPR possui características psicométricas satisfatórias e que pode ser usado para medir a personalidade resistente (desafio, controle e envolvimento) em adultos mexicanos.

Palavras-chave: personalidade resistente, adaptação, validação psicométrica, população mexicana.

\section{Introducción}

La personalidad resistente es un constructo que mediatiza el proceso salud-enfermedad, en este intervienen los comportamientos, las creencias, las actitudes y los valores entre otros componentes subjetivos asociados a las estrategias utilizadas para enfrentar las exigencias y retos del entorno sociocultural y ambiental. La personalidad resistente se ha vinculado al uso de herramientas cognitivas, actitudinales y comportamentales asociadas con el bienestar, el equilibrio y la salud integral; mientras que el uso de estrategias de afrontamiento evitativas o emocionales se ha relacionado con mayor vulnerabilidad a enfermar (Moreno-Jiménez et al., 2014; Menezes y Morán, 2016; Grau-Valdés et al., 2020).

El concepto de "hardiness" o "hardy personality" fue propuesto por primera vez por Suzanne Kobasa (1979) como un recurso personal contra los efectos negativos o estresantes en la salud y, específicamente, como un amortiguador del estrés laboral (Kobasa, 1982; Kobasa et al., 1982). Desde entonces, el constructo ha despertado mucho interés y esfuerzos para aclarar su contenido, evaluación y posibles efectos (Beehr y Bowling, 2005; Maddi, 2006; Maddi y Martínez, 2008; Maddi, 2013; Peñacoba y Moreno-Jiménez, 1998).

La propuesta de Kobasa se fundamenta en tres bases conceptuales: el modelo de diferencias individuales en las respuestas al estrés (Selye, 1956), el modelo de Lazarus (1966) sobre los mecanismos de evaluación del estrés, y las contribuciones de autores como Allport (1955) sobre los beneficios de algunas disposiciones de personalidad en el proceso de estrés (Kobasa, 1979).

Kobasa conceptualizó la resistencia como una variable de personalidad (Kobasa, 1979; Kobasa et al., 1982; Kobasa et al., 1983; Kobasa et al., 1985). El concepto de personalidad resistente tal como lo propone Kobasa queda definido por tres dimensiones, a saber: 
- Compromiso (commitment): consiste en el reconocimiento de las metas personales, así como la autoevaluación de las capacidades propias para tomar decisiones y mantenerse firme en los valores. Las personas con esta característica poseen tanto las habilidades como el deseo de enfrentarse a las situaciones de estrés de su día a día (Peñacoba y Moreno-Jiménez, 1998; Peñacoba y Moreno-Jiménez, 2000; Moreno-Jiménez et al., 2000a). También hace referencia al involucramiento de la persona en las actividades de su vida, el cual le permite encontrar un mayor sentido a las cosas y a los acontecimientos que ocurren en su entorno (Kobasa et al., 1982). Proporciona a la persona una serie de propósitos que ayudan a disminuir la amenaza percibida de cualquier estímulo estresante.

- Control: es la disposición a pensar y actuar con la convicción de que se puede intervenir en el curso de los acontecimientos. Los individuos con esta característica sienten que pueden manejar los estímulos estresantes en su propio beneficio, lo que posibilita que sean capaces de presentir consecuencias positivas en muchos de estos acontecimientos.

- Reto (challenge): permite a las personas percibir el cambio como una oportunidad y no como una amenaza, lo que proporciona flexibilidad cognitiva y tolerancia a la ambigüedad, que les induce a considerar los cambios como algo habitual en la vida y en la historia (Kobasa, 1982; Moreno-Jiménez et al., 2000a). Además, las personas con esta característica no se asustan ante las situaciones novedosas o estresantes, sino que las viven como una oportunidad para crecer y aprender nuevas cosas (Sheard y Golby, 2010).

Es importante resaltar que para que una persona sea considerada "resistente" debe poseer las tres características (Kobasa, 1979; Maddi, 2006; Moreno-Jiménez et al., 2000b).

Debe destacarse que existen diferencias entre el constructo de resiliencia y el de personalidad resistente. La personalidad resistente hace alusión a un macro factor de personalidad, compuesto por las dimensiones compromiso, desafío y control (Kobasa, 1979), por otra parte, la resiliencia puede contener estas dimensiones, pero no de modo exclusivo. Es decir, la hardiness emerge como un patrón de actitudes y comportamientos que mejora la resiliencia del sujeto ante situaciones potencialmente estresantes, constituyendo tan solo una de las posibles vías de desarrollo de resiliencia (Bonanno, 2004; Maddi, 2005; Maddi et al., 2012). De esta forma, aquellas personas que alcancen niveles elevados de hardiness tendrán una mayor probabilidad de ser resilientes (Fernández-Lansac y Crespo, 2011). 
Rosa M. Meda-Lara, Carmen Yeo-Ayala, Pedro Juárez-Rodríguez, Andrés Palomera-Chávez, 5 Bernardo Moreno-Jiménez

En el modelo de hardiness, la respuesta al estrés no es automática y lineal, sino el resultado de la interacción de un individuo con las fuentes del estrés. Según Oullette y Di Placido (2001), "se dice que la dureza disminuye los efectos negativos del estrés" (p. 178). Se han propuesto dos mecanismos principales para explicar este efecto, una percepción diferente de los eventos (Allred y Smith, 1989) y un uso más efectivo de las estrategias de afrontamiento (Ford-Gilboe y Cohen, 2000). Este tipo de afrontamiento ha sido considerado como "afrontamiento transformacional", caracterizado por una reinterpretación positiva de los eventos (Hystad et al., 2011).

Mientras que el modelo teórico de resistencia ha sido bien recibido e incorporado a los modelos de los efectos de la personalidad en los procesos generales de adaptación (Allport, 1955; Caprara y Cervone, 2000; Malkin et al., 2019), especialmente en respuesta al estrés (Abdollahi et al., 2016; Contrada y Guyll, 2001; Lazarus, 1999; Vollrath, 2006; Williams et al., 2011), los instrumentos para medir la resistencia han sido criticados debido a la falta de rigor metodológico.

Los componentes propuestos del constructo de resistencia no fueron el resultado de estudios empíricos, sino de un modelo teórico de la persona como actor activo que elige sus estrategias y objetivos (Maddi y Kobasa, 1981). En relación con los instrumentos que se han diseñado y validado para medir la personalidad resistente, el primero de ellos se basó en una serie de escalas preexistentes (Kobasa, 1979) y no fue apoyado por los resultados psicométricos. Sin embargo, el interés en el constructo ha promovido diversos intentos de desarrollo de instrumentos para medirlo (Maddi et al., 2006; Peñacoba y Moreno-Jiménez, 1998). En algunos casos, se han utilizado escalas improvisadas (Kuo y Tsai, 1986), en otros, escalas descartadas (Nakano, 1990) y aunque ahora se están utilizando instrumentos más avanzados, como el Personal Views Survey III (Maddi et al., 2006) y la escala de resiliencia disposicional (Bartone, 2006), todavía existen algunos problemas metodológicos, agravados por problemas para acceder libremente a los instrumentos. Como algunos autores han referido (Chan, 2000; Ghorbani et al., 2000; Sinclair y Tetrick, 2000), la investigación sobre la personalidad resistente ha estado fuertemente cargada con problemas generales de metodología, instrumentos y muestras.

Bajo el supuesto de que la resistencia no es solo una variable de personalidad general, sino que puede tomar formas específicas en determinadas áreas de comportamiento, las escalas de resistencia se han desarrollado para sectores específicos como la salud (Silva-Junior et al., 2020), el ámbito académico (Weigold et al., 2016), la cognición (Matthews et al, 2019), la resistencia militar (Adler y Dolan, 2006; Wang et al., 2019), la psicología deportiva (González-García y Pelegrín, 2020; Ponce-Carbajal et al., 2020; Serrato, 2017), los profesionales de la salud (Faílde-Garrido et al., 2013), las 
mujeres en situación de riesgo social y de salud (Bahrami et al., 2018; Barra y Vaccaro, 2013; Shifren et al., 2015), los cuidadores (Fernández-Lansac y Crespo, 2011), los bomberos (Pollet et al., 2016), el bornout (Moreno-Jiménez et al., 2006), la resistencia y la autoeficacia en estudiantes (Jie-Tsuen, 2015) o incluso para idiomas específicos, como el noruego (Hystad et al., 2011), en respuesta a la necesidad de considerar las formas culturales.

En España, se ha construido una línea de investigación centrada en la resistencia en el lugar de trabajo, con varias investigaciones y publicaciones (Garrosa et al., 2011; Garrosa et al., 2010; Ladsttäter et al., 2010; Moreno-Jiménez et al., 2006; Moreno-Jiménez et al., 2000a; Moreno-Jiménez et al., 2012; Peñacoba y MorenoJiménez, 1998; Peñacoba y Moreno-Jiménez, 2000). A partir de esta, se desarrolló el cuestionario de personalidad resistente (CPR) siguiendo las pautas internacionales para el desarrollo de escalas (Hambleton, 1980; Martínez et al., 2005; Morales, 2006). La evaluación psicométrica con el rigor metodológico correspondiente facilita la investigación sobre la hardiness y sus aplicaciones en el idioma castellano, asimismo, puede conducir a nuevas formulaciones y aplicaciones (Moreno-Jiménez et al., 2014).

En Chile, Carmona et al. (2017) realizaron una adaptación del CPR al contexto universitario, los análisis de factoriales confirmatorios reportaron un modelo con adecuados índices en todos los parámetros seleccionados $\left(X^{2}=220,57\right.$; RMSEA $=0,06$; $\mathrm{NFI}=0,91 ; \mid \mathrm{FI}=0,94 ; \mathrm{TLI}=0,93 ; \mathrm{y} \mathrm{CFI}=0,94)$. En este modelo están presentes los tres componentes de la personalidad resistente: control, implicación y reto. Este grupo reportó índices de consistencia interna adecuados, similares a lo obtenido en el CPR original (Moreno-Jiménez et al., 2000b) y superiores a lo reportado en otros trabajos (Maddi et al., 2006; Hystad et al., 2011; Moreno-Jiménez et al., 2014). En el análisis de correlación ítem-total observaron relaciones importantes según el estándar propuesto por Cohen (1992). Finalmente, presentaron relaciones estadísticamente significativas entre el CPR y los constructos de satisfacción vital, compromiso académico y los rasgos de personalidad en la dirección teórica esperada y observada previamente en otros estudios (Nayyeri y Aubi, 2011; Zhang, 2011; Pereira, 2013).

En Cuba, Grau-Valdéz et al. (2020) realizaron la adaptación y validación psicométrica del CPR en versión no laboral, este grupo de investigación reportó un modelo trifactorial (control, implicación y reto) con un factor de segundo orden (personalidad resistente) con índices de ajuste aceptables. Además, encontraron valores de confiabilidad admisibles, tanto global $(a=.83)$ como en cada dimensión: control y reto $(a=$ .77) e implicación $(a=.70)$. La validez concurrente aportó evidencia sobre el valor de las subescalas control y compromiso. 
Rosa M. Meda-Lara, Carmen Yeo-Ayala, Pedro Juárez-Rodríguez, Andrés Palomera-Chávez, 7 Bernardo Moreno-Jiménez

El propósito de este estudio fue adaptar y validar psicométricamente el CPR en una muestra de adultos mexicanos. Cabe mencionar que algunos de los reactivos fueron adaptados a un lenguaje común a los mexicanos y fueron redactados en un contexto general y no únicamente laboral.

\section{Material y método}

\section{Participantes}

La muestra fue incidental y el total de participantes fue de 357 adultos "relativamente sanos" sin diagnóstico de enfermedades crónicas, todos alfabetizados (sabían leer y escribir); la media de edad fue de 32,35 (DE=11,85) años cumplidos; el 63,3\% ( $n=226)$ fueron mujeres; el 66,4\% ( $n=237)$ vive con pareja estable; el 57,1 \% $(n=204)$ tienen una carrera profesional; el 18,5\% $(n=66)$ preparatoria y el 24,4\% restante, primaria, secundaria y/o carrera técnica. El 59,1 \% ( $n=211)$ trabajaba en el momento de la aplicación de las escalas. Todos los participantes firmaron consentimiento informado que gurada su anonimato.

\section{Instrumentos}

1. Cuestionario sociodemográfico. Requirió edad, estado civil, escolaridad, y ocupación de los participantes; además, se les pidió que contestaran a la pregunta: ¿padece algún problema de salud?

2. Cuestionario de personalidad resistente (Moreno-Jiménez et al., 2014). Consta de 15 reactivos en escala Likert con opciones de respuestas que van de "totalmente en desacuerdo" =1, "en desacuerdo" =2, "de acuerdo" =3, y "totalmente en desacuerdo" $=4$. El cuestionario fue diseñado para medir los componentes de implicación, control y reto de la personalidad resistente. El cuestionario fue desarrollado y validado en el contexto laboral; sin embargo, para la versión mexicana se hicieron ajustes en la redacción de alguno de los reactivos. Por ejemplo, en reactivo 1 se cambió el verbo "me implico" por el verbo "me involucro", en el reactivo 2, se modificó "elijo los trabajos que suponen para mí una nueva experiencia, aunque requiera mayor esfuerzo". El reactivo 3 "hago todo lo que puedo para asegurarme de tener control", el reactivo 5 "en mi vida me atraen preferentemente las nuevas experiencias" $\mathrm{y}$ los reactivos 10, 11, 12, 13, 14 y 15 fueron adaptados a la vida cotidiana. Se obtuvieron los coeficientes alfa de Cronbach de las 
escalas de implicación $=.85$, reto $=.84$, control $=.85$ y personalidad resistente global $=.93$.

3. Escala de satisfacción con la vida (Satisfaction With Life Scale, SWLS) (Diener et al., 1985). Se retoma la adaptación española (Atienza et al., 2000), con ligeras modificaciones para mayor claridad en México, por ejemplo, el primer ítem "En la mayoría de los aspectos mi vida es como quiero que sea" fue modificado a: "mi vida, en casi todo, responde a lo que aspiro". Los otros ítems que formaron la SWLS fueron: "las condiciones de mi vida son buenas"; "estoy satisfecho con mi vida"; "hasta ahora en mi vida he logrado cosas que eran importantes para mí"; y "si volviese a nacer, cambiaría bastantes cosas en mi vida" (calificación inversa). En cuanto a las opciones de respuesta, la Escala Likert en siete opciones: "no, en absoluto", "no, apenas", "más bien no", "ni sí ni no", "más bien sí", "sí, bastante", "sí, del todo". Los puntajes van de 1 a 7 . Los coeficientes alfa de Cronbach reportados van de $a=.79$ a $a=.89$ (Atienza et al., 2000; Blanco y Salazar, 2014; Amezquita et al., 2008); en este estudio fue $a=.88$.

4. Prueba de orientación vital (Life Orientation Test, LOT) (Scheier et al., 1994) consta de 10 ítems en escala Likert de 5 puntos (siempre, casi siempre, a veces, casi nunca y nunca), 6 ítems miden la dimensión de optimismo disposicional, en tanto que los otros 4 ítems son distractores denominados así por el autor. De los 6 ítems, 3 están redactados en sentido positivo (dirección optimista), y 3 en sentido negativo (dirección pesimista), en diferentes estudios el uso de la prueba ha arrojado valores del coeficiente alfa de Cronbach .71 y relaciones entre ítem-total entre .40 a .70. Estudios con población española muestran coeficientes alfa de .75 (Remor et al., 2006). En la adaptación al castellano original, el coeficiente alfa de Cronbach fue de .78 y la correlación con el LOT original fue de .95 (Otero et al., 1998). En este estudio se utiliza una adaptación de 5 reactivos con un $a=.80$.

5. Escala de autoeficacia general (Baessler y Schwarzer, 1996). Evalúa el sentimiento estable de competencia personal para manejar de forma eficaz una gran variedad de situaciones estresantes. Se utilizó la versión propuesta por Pérez-García et al. (2000); que consta de 10 ítems con escalas de respuesta de 10 puntos. Se realizó una adaptación para México de 5 reactivos. Las confiabilidades alfa de Cronbach reportadas en la consistencia interna obtenida $(a=.87)$. En este estudio fue $a=.91$.

6. Cuestionario general de salud (General Health Questionnaire, GHQ) (Goldberg y Williams, 1988). Es un cuestionario que mide la evaluación que hace el individuo de su estado de bienestar general, especialmente en lo 
Rosa M. Meda-Lara, Carmen Yeo-Ayala, Pedro Juárez-Rodríguez, Andrés Palomera-Chávez, 9 Bernardo Moreno-Jiménez

que se refiere a la presencia de ciertos estados emocionales. Para este estudio se utilizaron las subescalas de Ansiedad y trastornos del sueño que constan de 7 reactivos en escala Likert con cuatro opciones de respuestas que van de no, en absoluto $=1$, no más de lo habitual $=2$, bastante más de lo habitual $=3$ y mucho más que lo habitual $=4$. Se obtuvo un $a=.88$ en esta escala; y la subescala de disfunción social que consta de 7 reactivos en escala Likert con cuatro opciones de respuestas que van de más activo que lo habitual = 1; igual que lo habitual $=2$; bastante menos que lo habitual $=3 \mathrm{y}$ mucho menos que lo habitual=4. Se obtuvo un $a=.74$ en esta escala.

\section{Procedimiento}

Los instrumentos, el cuestionario sociodemográfico y una carta de consentimiento informado fueron convertidos a formato electrónico a través del software SurveyMonkey. Posteriormente, los cuestionarios fueron enviados a la dirección de correo electrónico de potenciales participantes, adultos de entre 18 y 60 años que residían en los estados de Jalisco, Michoacán, Colima y Ciudad de México. Los datos fueron obtenidos en el periodo comprendido de agosto de 2016 a enero de 2017.

El proyecto fue aprobado por el Consejo de Ética del Centro Universitario de Ciencias de la Salud de la Universidad de Guadalajara. Además, fueron tomados en consideración la Declaración de Helsinki de 2004 y el Código Ético del Psicólogo (Sociedad Mexicana de Psicología, 2007), cuidando la integridad de los participantes y haciendo un uso confidencial de los datos.

\section{Análisis estadístico}

Los análisis fueron realizados usando el paquete estadístico SPSS ${ }^{\circledR} 23$, con el que se obtuvieron estadísticos descriptivos (media, desviación estándar) para conocer la distribución, el coeficiente alfa de Cronbach para evaluar la consistencia interna y análisis de correlaciones de Pearson.

El análisis factorial confirmatorio se realizó con el programa Mplus 7 (Muthén y Muthén, 2012). Para la realización del análisis factorial se comprobó la normalidad de la población con la prueba contraste bilateral de Mardia de asimetría y curtosis multivariada, (Wang y Wang, 2012) y al encontrarse violación de la normalidad ( $p \leq$ .001), se empleó el método de máxima verosimilitud en su versión robusta (MLR), por lo que los índices de ajuste que se utilizaron fueron el Comparative Fit Index (CFI), el Tucker-Lewis Index (TLI), con valores iguales o mayores a .90 y el Standardized 
Root Mean Square Residual (SRMR) y el Root Mean Squared Error of Approximation (RMSEA), con valores iguales o menores a .08 (Wang y Wang, 2012).

Para la validez del constructo se realizaron análisis de correlación de la personalidad resistente con las variables de autoeficacia, satisfacción con la vida y optimismo.

\section{Resultados}

\section{Descripción de la muestra}

Se obtuvieron datos confiables de 357 adultos relativamente sanos, todos mexicanos, cuya edad osciló entre 18 y 64 años $(M=33,42, D E=11,67)$. Predominaron las mujeres con el 60 \%; en cuanto a su relación de pareja un poco más del 60 \% tenían pareja en el momento del estudio; en cuanto a si tenían hijos se distribuyeron homogéneamente casi con el 50 \% los que tenían y los que no. Por nivel de estudios predomina los que tienen formación universitaria con el 57 \% y preparatoria 19,5 \% (ver tabla 1).

Tabla 1. Datos sociodemográficos de la muestra total

\begin{tabular}{|c|c|c|}
\hline Variable & $M / D E$ & n (\%) \\
\hline \multicolumn{3}{|l|}{ Sexo } \\
\hline Mujer & & $226(63,3)$ \\
\hline Hombre & & $131(36,7)$ \\
\hline Edad & $33,42 / 11,67$ & \\
\hline \multicolumn{3}{|l|}{ Relación } \\
\hline & Con pareja actualmente & $237(66,4)$ \\
\hline & Sin pareja actualmente & $120(33,6)$ \\
\hline \multicolumn{3}{|l|}{ Hijos } \\
\hline & Con hijos & $174(48,7)$ \\
\hline & Sin hijos & $183(51,3)$ \\
\hline \multicolumn{3}{|l|}{ Nivel de estudios } \\
\hline & Saben leer y escribir & $2(0,6)$ \\
\hline & Primaria & $20(5,6)$ \\
\hline & Secundaria & $36(10,1)$ \\
\hline & Carrera técnica & $29(8,1)$ \\
\hline & Preparatoria & $66(19,5)$ \\
\hline & Universitaria & $204(57,1)$ \\
\hline \multicolumn{3}{|l|}{ Trabaja actualmente } \\
\hline & $\mathrm{Si}$ & $211(59,1)$ \\
\hline & No & $146(40,9)$ \\
\hline
\end{tabular}

Fuente: elaboración propia. 


\section{Análisis descriptivos y correlaciones de los ítems del cuestionario de personalidad resistente}

En la tabla 2 se presenta el análisis descriptivo y las correlaciones entre los reactivos del cuestionario de personalidad resistente en la muestra de mexicanos sin afectaciones de salud. Se observa que todos los ítems del CPR se correlacionaron positivamente entre sí como era de esperarse.

Tabla 2. Medias, desviaciones estándar y correlaciones de los ítems del cuestionario de personalidad resistente

\begin{tabular}{|c|c|c|c|c|c|c|c|c|c|c|c|c|c|c|c|c|}
\hline & $M$ & SD & 1 & 2 & 3 & 4 & 5 & 6 & 7 & 8 & 9 & 10 & 11 & 12 & 13 & 14 \\
\hline Ítem 1 & 3,32 & 0,74 & 1 & & & & & & & & & & & & & \\
\hline Ítem 2 & 3,09 & 0,80 & $0,637^{\star \star}$ & 1 & & & & & & & & & & & & \\
\hline Ítem 3 & 2,98 & 0,77 & $0,549^{\star *}$ & $0,461^{\star *}$ & 1 & & & & & & & & & & & \\
\hline Ítem 4 & 3,11 & 0,77 & $0,524^{\star \star}$ & $0,505^{\star \star}$ & $0,564^{\star \star}$ & 1 & & & & & & & & & & \\
\hline Ítem 5 & 3,05 & 0,78 & $0,437^{\star *}$ & $0,503^{* *}$ & $0,385^{* \star}$ & $0,399^{* *}$ & 1 & & & & & & & & & \\
\hline Ítem 6 & 3,25 & 0,75 & $0,518^{\star *}$ & $0,383^{* *}$ & $0,537^{\star \star}$ & $0,472^{* *}$ & $0,367^{\star *}$ & 1 & & & & & & & & \\
\hline Ítem 7 & 3,13 & 0,78 & $0,593^{\star *}$ & $0,473^{* *}$ & $0,514^{* *}$ & $0,610^{* *}$ & $0,365^{* *}$ & $0,596^{\star *}$ & 1 & & & & & & & \\
\hline Ítem 8 & 3,12 & 0,80 & $0,539^{\star \star}$ & $0,578^{* *}$ & $0,527^{\star *}$ & $0,509^{\star *}$ & $0,548^{\star *}$ & $0,489^{* *}$ & $0,602^{* *}$ & 1 & & & & & & \\
\hline Ítem 9 & 2,87 & 0,80 & $0,337^{\star *}$ & $0,291^{\star *}$ & $0,525^{* *}$ & $0,363^{* *}$ & $0,232^{* *}$ & $0,526^{* *}$ & $0,412^{* *}$ & $0,473^{\star *}$ & 1 & & & & & \\
\hline Ítem 10 & 3,06 & 0,74 & $0,485^{\star *}$ & $0,412^{* *}$ & $0,452^{* *}$ & $0,470^{\star \star}$ & $0,267^{* *}$ & $0,475^{* *}$ & $0,595^{* *}$ & $0,491^{\star \star}$ & $0,437^{\star *}$ & 1 & & & & \\
\hline Ítem 11 & 3,05 & 0,77 & $0,448^{\star \star}$ & $0,531^{\star *}$ & $0,464^{* *}$ & $0,451^{\star \star}$ & $0,641^{* *}$ & $0,463^{* *}$ & $0,439^{* *}$ & $0,581^{\star \star}$ & $0,386^{* *}$ & $0,424^{* *}$ & 1 & & & \\
\hline Ítem 12 & 3,26 & 0,72 & $0,517^{\star \star}$ & $0,365^{\star *}$ & $0,514^{* *}$ & $0,469^{\star \star}$ & $0,244^{* *}$ & $0,666^{\star *}$ & $0,552^{* *}$ & $0,456^{\star *}$ & $0,557^{\star *}$ & $0,451^{* *}$ & $0,464^{* *}$ & 1 & & \\
\hline Ítem 13 & 3,00 & 0,77 & $0,334^{* *}$ & $0,448^{* *}$ & $0,393^{\star *}$ & $0,413^{* *}$ & $0,583^{* *}$ & $0,401^{* *}$ & $0,392^{\star *}$ & $0,535^{\star *}$ & $0,378^{* *}$ & $0,377^{\star \star}$ & $0,735^{* *}$ & $0,386^{* *}$ & 1 & \\
\hline Ítem 14 & 3,25 & 0,74 & $0,436^{* *}$ & $0,347^{\star *}$ & $0,506^{\star \star}$ & $0,395^{* *}$ & $0,272^{\star *}$ & $0,584^{\star *}$ & $0,498^{\star *}$ & $0,493^{* *}$ & $0,474^{* *}$ & $0,559^{* *}$ & $0,484^{* *}$ & $0,613^{* *}$ & $0,470^{* *}$ & 1 \\
\hline Ítem 15 & 3,11 & 0,74 & $0,446^{\star *}$ & $0,321^{\star *}$ & $0,489^{* *}$ & $0,411^{\star *}$ & $0,253^{* *}$ & $0,572^{* *}$ & $0,501^{\star *}$ & $0,446^{\star *}$ & $0,607^{* *}$ & $0,459^{* *}$ & $0,418^{* *}$ & $0,653^{* *}$ & $0,379^{* *}$ & $0,610^{* *}$ \\
\hline
\end{tabular}

Fuente: elaboración propia. 


\section{Análisis factorial confirmatorio del cuestionario de personalidad resistente}

En el análisis factorial confirmatorio se comprobó el ajuste de dos modelos, uno con 15 ítems agrupados en un solo factor y otro trifactorial (implicación, control y reto) con un factor de segundo orden (personalidad resistente). El primer modelo solo mostró ajuste en el SRMR, mientras que el segundo modelo se ajustó adecuadamente en los 4 índices considerados (ver tabla 3).

Tabla 3. Índices de ajuste para los modelos del cuestionario de personalidad resistente

\begin{tabular}{lcccc}
\hline & RMSEA & CFI & TLI & SRMR \\
\hline Un factor & 0,090 & 0,885 & 0,886 & 0,055 \\
\hline Tres factores y uno de segundo orden* & 0,074 & 0,925 & 0,909 & 0,046 \\
\hline
\end{tabular}

* Modelo con valores de ajuste más adecuado

Fuente: elaboración propia.

En cuanto a los análisis de fiabilidad se encontraron valores adecuados tanto de la escala en conjunto $(a=0,93)$, como en los tres factores: implicación $(a=0,84)$, reto $(a=0,86)$ y control $(a=0,86)$.

En la figura 1 se muestra el modelo de tres factores y uno de segundo orden con sus respectivas cargas factoriales y error estándar, así como las $\beta$ del constructo de personalidad resistente con implicación $(0,90)$, reto $(0,80)$ y control $(0,89)$. 


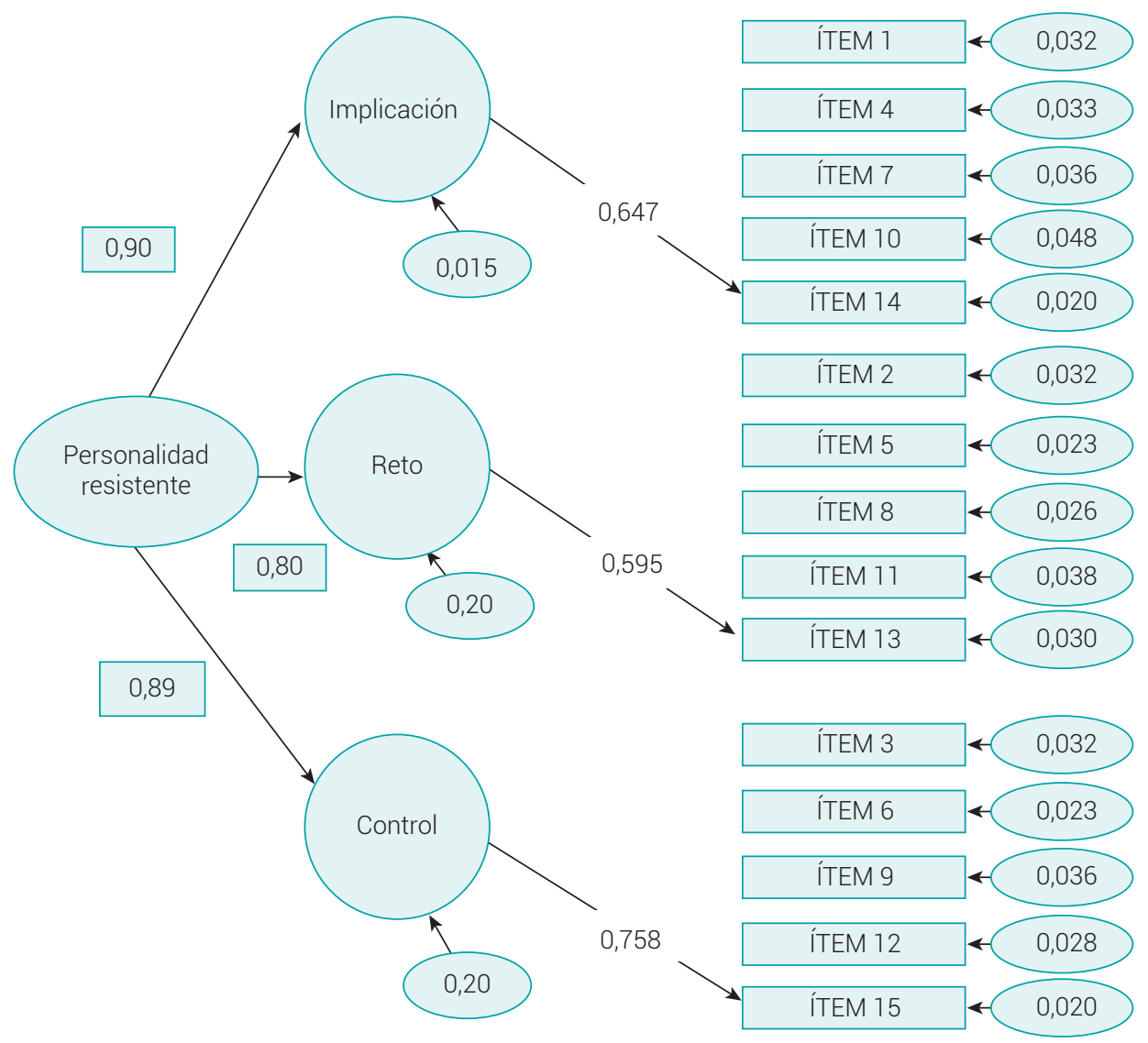

Figura 1. Análisis factorial confirmatorio del CPR Fuente: elaboración propia.

\section{Validación del constructo}

Para determinar la validez del constructo, se realizó análisis de correlación de orden cero entre las dimensiones del CPR y otras variables psicológicas teóricamente relacionadas. Como se puede observar en la tabla 4, las dimensiones de implicación, reto y control se correlacionaron positivamente con las variables de optimismo, satisfacción con la vida y autoeficacia $(p \leq 0,01)$ en todos los casos, tal como se esperaba. Asimismo, como era de esperar, los componentes de implicación y reto se correlacionaron negativamente con las variables negativas de ansiedad y trastornos del sueño y disfunción social, a excepción de control que no se relacionó con ansiedad. Estas correlaciones fueron en la dirección esperada y respaldan la validez del CPR. También en la tabla 4 se presentan los coeficientes alfa de Cronbach para cada una de las dimensiones de la personalidad resistente y variables relacionadas que van de 0,80 a 0,91 . 
Tabla 4. Descriptivos, confiabilidad y correlaciones entre las variables estudiadas

\begin{tabular}{|c|c|c|c|c|c|c|c|c|c|c|}
\hline Variable & M & DE & $\boldsymbol{\alpha}$ & 1 & 2 & 3 & 4 & 5 & 6 & 7 \\
\hline 1. Implicación & 15,90 & 2,88 & 0,85 & & & & & & & \\
\hline 2. Control & 15,48 & 2,98 & 0,86 & $0,762^{* *}$ & & & & & & \\
\hline 3. Reto & 15,33 & 2,86 & 0,86 & $0,752^{\star *}$ & $0,672^{\star *}$ & & & & & \\
\hline 4. Autoeficacia & 43,24 & 6,52 & 0,91 & $0,253^{* *}$ & $0,185^{* *}$ & $0,239 \cdots \cdot$ & & & & \\
\hline 5. Optimismo & 19,63 & 3,23 & 0,80 & $0,380^{* *}$ & $0,285^{\star *}$ & $0,333^{* *}$ & $0,308^{* *}$ & & & \\
\hline 6. Satisfacción con la vida & 26,09 & 5,92 & 0,88 & $0,344^{* *}$ & $0,194^{\star \star}$ & $0,290^{* *}$ & $0,428^{* *}$ & $0,535^{\star *}$ & & \\
\hline 7. Ansiedad y Trastornos del sueño & 11,98 & 4,43 & 0,88 & $-0,187^{\star \star}$ & $-0,007$ & $-0,198^{\star \star}$ & $-0,347^{\star *}$ & $-0,296^{\star *}$ & $-0,437^{\star \star}$ & \\
\hline 8. Disfunción Social & 13,17 & 2,54 & 0,74 & $-0,275^{\star *}$ & $-0,205^{\star *}$ & $-0,293^{\cdots} \cdot$ & $-0,407^{\star *}$ & $-0,454^{\star *}$ & $-0,399^{\star *}$ & $0,361^{* *}$ \\
\hline
\end{tabular}

**. La correlación es significativa en el nivel 0,01 (bilateral)

Fuente: elaboración propia.

\section{Discusión}

Ante la limitación de no contar con instrumentos psicométricos confiables para evaluar la personalidad resistente en idioma castellano y específicamente para población adulta mexicana, nuestros resultados sugieren que la adaptación del CPR (Moreno-Jiménez et al., 2014) tiene las propiedades psicométricas adecuadas para ser utilizado en población mexicana.

Los resultados del análisis factorial confirmatorio sugieren un modelo de tres factores con valores de ajuste adecuados: implicación, control y reto, que se relacionan con el constructo de la personalidad resistente. Esta estructura es consistente con lo reportado por otros autores (Sinclair y Tetrick, 2000; Maddi et al., 2006; Moreno-Jiménez et al., 2014, Carmona-Halty et al., 2017; Grau-Valdés et al., 2020 y Luceño-Moreno et al., 2020).

Se obtuvieron índices de consistencia interna adecuados en los componentes del CPR, de forma similar a los reportados por otros autores (Moreno-Jiménez et al., 2000a; Moreno-Jiménez et al., 2014, Carmona-Halty, 2017, Luceño-Moreno et al., 2020). 
Rosa M. Meda-Lara, Carmen Yeo-Ayala, Pedro Juárez-Rodríguez, Andrés Palomera-Chávez, 15 Bernardo Moreno-Jiménez

En este trabajo se presentaron correlaciones positivas entre los componentes del CPR y variables psicológicas positivas como la autoeficacia (Vinaccia et al., 2005; Ríos et al., 2010), el optimismo (Maury-Ortiz et al., 2014) y la satisfacción con la vida (Gutiérrez-Caballero et al., 2019); así como las correlaciones negativas con la ansiedad y trastornos del sueño (Luceño-Moreno et al., 2020) y disfunción social (Ríos et al., 2010; Eschleman et al., 2010; Luceño-Moreno et al., 2020), de forma coincidente con la literatura científica sobre el tema. Solo el factor "control" no se correlacionó con la ansiedad y trastornos del sueño. De lo cual se deduce que las personas con niveles altos de "implicación", "control" y "reto" tienden a tener menores manifestaciones de afectos negativos tales como la ansiedad y la disfunción social.

Con base en los resultados, se propone al CPR como un instrumento de evaluación psicométricamente validado en población adulta mexicana, el cual puede ser utilizado para valorar la respuesta frente a eventos estresantes de la vida, las estrategias de afrontamiento centradas en el problema, la autoeficacia, el optimismo, la satisfacción con la vida, las competencias para amortiguar las situaciones de estrés, la sintomatología ansiosa y disfunción social en las personas. Desde este enfoque centrado en las fortalezas y los recursos puede considerarse la resistencia como una variable de la personalidad que proporciona respuestas positivas a los eventos adversos, en este sentido, se puede estimar dentro de la corriente actual de la psicología positiva (Maddi y Martínez, 2008). Según el primer modelo propuesto por Kobasa (1979) y más recientemente por Maddi (2006), la resistencia psicológica puede considerarse un proceso de evaluación cognitiva, una perspectiva positiva de la vida y una línea de acción multifacética. Oullette y Di Placido (2001) lo han considerado como una forma distintiva y activa de entender las relaciones de uno con los demás, con los objetivos y con los problemas.

La investigación realizada en el idioma castellano con el CPR muestra que la personalidad resistente se puede presentar como un constructo de tres componentes con una buena consistencia interna desde una perspectiva psicométrica.

\section{Limitaciones del estudio}

La selección de la muestra fue incidental, por lo que no hubo un control azaroso; sin embargo, los participantes se autopercibían como "relativamente sanos", ya que no contaban con diagnóstico clínico de enfermedades crónicas o infecto contagiosas. 


\section{Referencias}

Abdollahi, A., Abu Talib, M., Carlbring, P., Harvey, R., Yaacob, S. N., y Ismail, Z. (2016). Problemsolving skills and perceived stress among undergraduate students: The moderating role of hardiness. Journal of Health Psychology, 23(10), 1321-1331. 10.1177/1359105316653265

Adler, A. B. y Dolan, C. A. (2006). Military hardiness as a buffer of psychological health on return from deployment. Military Medicine, 171(2), 93-98. 10.7205/milmed.171.2.93

Allred, K. D. y Smith, T. W. (1989). The hardy personality: Cognitive and physiological responses to evaluative threat. Journal of Personality and Social Psychology, 56(2), 257-266. https://doi. org/10.1037/0022-3514.56.2.257

Allport, G. W. (1955). Becoming: Basic considerations for a psychology of personality. Yale University Press.

Amezquita, M., González, R. y Zuluaga, D. (2008). Prevalencia de depresión e ideación suicida en estudiantes de $8^{\circ}, 9^{\circ}, 10^{\circ}$ y $11^{\circ}$ grado, en ocho colegios oficiales de Manizales. Hacia la Promoción de la Salud, 13, 143-153.

Atienza, F., Pons, D., Balaguer, I. y García-Merita, M. (2000). Propiedades Psicométricas de la Escala de Satisfacción con la Vida en Adolescentes. Psicothema, 12(2), 314-319.

Baessler, J. y Schwarzer, R. (1996). Evaluación de la autoeficacia: adaptación española de la escala de autoeficacia general. Ansiedad y Estrés, 2(1), 1-7.

Bahrami, M., Mohamadirizi, S. y Mohamadirizi, S. (2018). Hardiness and optimism in women with breast cancer. Iranian Journal of Nursing and Midwifery Research, 23(2), 105-110. doi: 10.4103/ ijnmr.IJNMR_200_16

Barra, E. y Vaccaro, M. A. (2013). Estrés percibido, afrontamiento y personalidad resistente en mujeres infértiles. Liberabit Revista Peruana de Psicología, 19(1), 113-119.

Bartone, P. T. (2006). Resilience under military operational stress: Can leaders influence hardiness? Military Psychology, 18(Suppl. 1), S131S148. https://doi.org/10.1207/s15327876mp1803s_10

Beehr, T. A. y Bowling, N. A. (2005). Hardy personality, stress, and health. En: C. L. Cooper (ed.), Handbook of stress medicine and health (2a. ed., pp. 193-211). CRC Press.

Blanco, M. y Salazar, M. (2014). Escala de satisfacción con la vida para adultos mayores de Emmons, Larsen y Griffin. En: V. Smith-Castro (comp.), Compendio de instrumentos de medición IIP2014, (pp. 227-231). Universidad de Costa Rica. 
Bonanno, G. (2004). Loss, trauma, and human resilience: Have we underestimated the human capacity to thrive after extremely aversive events? American Psychologist, 59(1), 20-28. 10.1037/0003-066X.59.1.20

Caprara, G. V. y Cervone, D. (2000). Personality: Determinants, dynamics, and potentials. Cambridge University Press.

Carmona-Halty, M., Garrosa, E. y Moreno-Jiménez, B. (2017). Análisis psicométrico de la Escala de Personalidad Resistente (EPR) adaptada a estudiantes universitarios chilenos. Interciencia, 42(5), 286-292.

Chan, D. W. (2000). Dimensionality of hardiness and its role in the stress distress relationship among Chinese adolescents in Hong Kong. Journal of Youth and Adolescence, 29, 147-161. 10.1023/A:1005100531194

Contrada, R. J. y Guyll, M. (2001). On who gets sick and why: The role of personality, stress, and disease. En, A. Baum, T. A. Revenson y J. E. Singer (eds.), Handbook of health psychology (pp. 59-81). Erlbaum.

Cohen,J.(1992).Apowerprimer.PsychologicalBulletin,112(1),155-159.10.1037/0033-2909.112.1.155

Diener, E., Emmons, R. A., Larsen, R. J. y Griffin, S. (1985). The Satisfaction with Life Scale. Journal of Personality Assessment, 49, 71-75. https://doi.org/10.1207/s15327752jpa4901_13

Eschleman, K. J., Bowling, N. A. y Alarcon, G. M. (2010). A meta-analytic examination of hardiness. International Journal of Stress Management, 17(4), 277-307. https://doi.org/10.1037/a0020476

Faílde-Garrido, J. M., Carballo, J. A., Lameiras, M. y Rodríguez, Y. (2013). Personalidad resistente, calidad de vida y burnout en profesionales de la salud de centros gerontológicos. Salud (i) Ciencia, 19(7), 599-606.

Fernández-Lansac, V. y Crespo L., M. (2011). Resiliencia, personalidad resistente y crecimiento en cuidadores de personas con demencia en el entorno familiar: una revisión. Clínica y Salud, 22(1), 21-40.

Ford-Gilboe, M.C. y Cohen, J.A., 2000. Hardiness: A model of commitment, challenge, and control. En Rice, H. (ed.), Handbook of stress, coping and health. Implications for nursing research, theory, and practice (pp. 425-436). Sage. 
Garrosa, E., Moreno-Jiménez, B., Rodríguez-Muñoz, A. y Rodríguez-Carvajal, R. (2011). Role stress and personal resources in nursing: A cross-sectional study of burnout and engagement. International Journal of Nursing Studies, 48(4), 479-489. 10.1016/j.ijnurstu.2010.08.004

Garrosa, E., Rainho, C., Moreno-Jiménez, B. y Monteiro M. J. (2010). The relationship between job stressors, hardy personality, coping resources and burnout in a sample of nurses: A correlational study at two time points. International Journal of Nursing Studies, 47(2), 205-215. 10.1016/j.ijnurstu.2009.05.014

Ghorbani, N., Watson, P. J. y Morris, R. J. (2000). Personality, stress and mental health: Evidence of relationships in a sample of Iranian managers. Personality and Individual Differences, 28(4), 647-657. 10.1016/S0191-8869(99)00128-2

Goldberg, D. y Williams, P. (1988). A user's guide to the General Health Questionnaire. NFER-Nelson.

González-García, H. y Pelegrín, A. (2020). Influence of Perceived Parental Education Styles on Hardy Personality in Sport. Journal of Sports Science and Medicine, 19, 158-165.

Grau-Valdés, Y., Oliva-Hernández, I., Rojas-Ricardo, L., Grau-Abalo, J. A. y Martinez-Rodríguez, L. (2020). Propiedades psicométricas del Cuestionario de Personalidad Resistente (versión no laboral) en la población cubana. Terapia Psicológica, 38(2), 53-167. 10.4067/ s0718-48082020000200153

Gutiérrez-Caballero, J. M., Blázquez-Manzano, A. y Feu, S. (2019). Resistencia ocupacional y satisfacción con la vida en maestros españoles. RELIEVE, 25(2), 1-13. 10.7203/relieve.25.2.13139

Hambleton, R. K. (1980). Test score validity and standard setting methods. En R. A. Berk (ed.), Criterion-referenced measurement: The state of the art, (pp. 80-123). John Hopkins University Press.

Hystad, S. W., Eid, J. y Brevik, J. I. (2011). Effects of psychological hardiness, job demands, and job control on sickness absence: A prospective study. Journal of Occupational Health Psychology, 16(3), 265-278. 10.1037/a0022904

Jie-Tsuen, H. (2015). Hardiness, perceived employability, and career decision self-efficacy among taiwanese college students. Journal of Career Development, 42(4), 311-324. 10.1177 /0894845314562960

Kobasa, S. C. (1979). Stressful life events, personality, and health: An inquiry into hardiness. Journal of Personality and Social Psychology, 37(1), 1-11. 10.1037/0022-3514.37.1.1 
Rosa M. Meda-Lara, Carmen Yeo-Ayala, Pedro Juárez-Rodríguez, Andrés Palomera-Chávez, 19 Bernardo Moreno-Jiménez

Kobasa, S. C. (1982). Commitment and coping in stress resistance among lawyers. Journal of Personality and Social Psychology, 42(4), 707-717. 10.1037/0022-3514.42.4.707

Kobasa, S. C., Maddi, S. R. y Kahn, S. (1982). Hardiness and Health: A prospective study. Journal of Personality and Social Psychology, 42(1), 168-177. 10.1037/0022-3514.42.1.168

Kobasa, S. C., Maddi, S. R. y Zola, M. A. (1983). Type A and hardiness. Journal of Behavioral Medicine, 6, 41-51. 10.1007/BF00845275

Kobasa, S. C., Maddi, S. R., Puccetti, M. C. y Zola, M. A. (1985). Effectiveness of hardiness, exercise, and social support as resources against illness. Journal of Psychosomatic Research, 29(5), 525-533. https://doi.org/10.1016/0022-3999(85)90086-8

Kuo, W. H. y Tsai, Y. M. (1986). Social networking, hardiness, and immigrants' mental health. Journal of Health and Social Behavior, 27(2), 133-149. https://doi.org/10.2307/2136312

Ladstätter, F., Garrosa, E., Badea, C. y Moreno-Jiménez, B. (2010). Application of artificial neural networks to a study of nursing burnout. Ergonomics, 53(9), 1085-1096. 10.1080/00140139.201 0.502251

Lazarus, R.S. (1966). Psychological stress and the coping process. McGraw-Hill.

Lazarus, R.S. (1999). Stress and emotion: A new synthesis. Springer.

Luceño-Moreno, L., Talavera-Velasco, B., Jaén-Díaz, M. y Martín-García, J. (2020). Hardy personality assessment: validating the Occupational Hardiness Questionnaire in police officers. Profesional Psychology: Research and Practice, 51(3), 297-303. 10.1037/pro0000285

Maddi, S. R. (2005). On hardiness and other pathways to resilience. American Psychologist, 60(3), 261-262. 10.1037/0003-066X.60.3.261

Maddi, S. R. (2006). Hardiness: The courage to be resilient. En J. C. Thomas y D. L. Segal (eds.), Comprehensive handbook of personality and psychopathology (pp. 396-321). Wiley.

Maddi, S. R. (2013). Hardiness: turning stressful circumstances into resilient growth. Springer. 10.1007/978-94-007-5222-1

Maddi, S. R., Harvey, R. H, Khoshaba, D. M, Lu, J. L., Persico, M. y Brown, M. (2006). The personality construct of hardiness. III: Relationships with repression, innovativeness, authoritarianism, and performance. Journal of Personality, 74(2), 575-598. 10.1111/j.1467-6494.2006.00385.x 
Maddi, S. R. y Kobasa, S. C. (1981). Intrinsic motivation and health. En H. I. Day (ed.), Advances in intrinsic motivation and aesthetics (pp. 120-133). Plenum Press.

Maddi, S. R. y Martínez, M. L. (2008). La personalidad resistente: promoviendo el crecimiento ante condiciones de estrés. En C. Vázquez y G. Hervás (eds.), Psicología positiva aplicada (pp. $217-$ 236). Desclee de Brouwer.

Maddi, S. R., Matthews, M. D., Kelly, D. R., Villarreal, B. y White, M. (2012). The role of hardiness and grit in predicting performance and retention of USMA cadets. Military Psychology, 24:19-28. doi: 10.1080/08995605.2012.639672

Malkin, V., Rogaleva, L., Kim, A. y Khon, N. (2019). The hardiness of adolescents in various social groups. Frontiers in Psychology, 10, 2427. 10.3389/fpsyg.2019.02427

Martínez, R., Moreno, R. y Muñiz, J. (2005). Construcción de los ítems. En J. Muñiz, M. A. Fidalgo, E. García-Cueto, R. Martínez, y R. Moreno, Análisis de los ítems, (pp. 9-52). La Muralla.

Matthews, G., Panganiban, A. R., Wells, A., Wohleber, R. W. y Reinerman-Jones, L. E. (2019). Metacognition, Hardiness, and Grit as Resilience Factors in Unmanned Aerial Systems (UAS) Operations: A Simulation Study. Frontiers in Psychology, 10, 640. 10.3389/fpsyg.2019.00640

Maury-Ortiz, J. G., Martínez-Lugo, M. E. y González-Colón, Z. L. (2014). Relación del optimismo, la personalidad resistente y el engagement con el trabajo en una muestra de empleados. Revista Puertorriqueña de Psicología, 25(2):280-296.

Menezes, E., y Morán, C. (2016). A personalidade resiliente: uma conceptualização teórica. International Journal of Developmental and Educational Psychology, 2(1), 151-156.

Morales, P. (2006). Medición de actitudes en psicología y educación. Construcción de escalas y problemas metodológicos. Universidad Pontificia de Comillas.

Moreno-Jiménez, B., Garrosa, E. y González, J. (2000a). Personalidad Resistente, Burnout y salud. Escritos de Psicología, 4, 64-77.

Moreno-Jiménez, B., Garrosa, E. y González, J. L. (2000b). Variables de personalidad y proceso del burnout. Personalidad resistente y sentido de coherencia. Revista Interamericana de Psicología Ocupacional, 20(1), 1-18.

Moreno-Jiménez, B., Garrosa, E., Corso, S., Boada, M. y Rodríguez-Carvajal, R. (2012). Personalidad resistente y capital psicológico: las variables personales positivas y los procesos de agotamiento y vigor. Psicothema, 24(1), 79-86. 
Moreno-Jiménez, B., Natera, N. I., Muñoz, A. y Benadero, M. E. (2006). La personalidad resistente como variable moduladora del síndrome de burnout: estudio en una muestra de bomberos. Psicothema, 18(3), 413-418.

Moreno-Jiménez, B., Rodríguez-Muñoz, A., Garrosa, H. E. y Blanco, L. M. (2014). Development and validation of the occupational hardiness questionnaire. Psicothema, 26(2), 207-214. 10.7334/ psicothema2013.49

Muthén, L. K. y Muthén, B. O. (2012). Mplus User’s Guide. (7a. edicón). Muthén \& Muthén.

Nakano, K. (1990). Hardiness, Type A behavior, and physical symptoms in a Japanese sample. Journal of Nervous and Mental Disease, 178(1), 52-56. 10.1097/00005053-199001000-00010

Nayyeri, M. y Aubi, S. (2011). Prediction well being on basic components of hardiness. Social and Behavioral Sciences, 30, 1571-1575. 10.1016/j.sbspro.2011.10.305

Otero, J. M., Luengo, A., Romero, E., Gómez, J. A. y Castro, C. (1998). Psicología de la personalidad. Manual de prácticas. Ariel Practicum.

Oullette, S. C. y Di Placido, J. (2001). Personality's role in the protection and enhancement of health: Where the research has been, where it is stuck, how it might move. En A. Baum, T. A. Revenson y J. E. Singer (eds.), Handbook of health psychology (pp. 175-193). Erlbaum.

Peñacoba, C.y Moreno-Jiménez, B. (1998). El concepto de personalidad resistente. Consideraciones teóricas y aplicaciones prácticas. Boletín de Psicología, 58, 61-96.

Peñacoba, C. y Moreno-Jiménez, B. (2000). La evaluación de la personalidad resistente y su influencia en la salud. Boletín de Psicología, 67, 45-64.

Pereira, M. (2013). Hardiness, engagement e satisfação com a vida em alunos de Academia Militar: relação entre variáveis e estudos comparativos. [Tesis, Universidad de Lisboa].

Pérez-García, A. M., Sanjuan, P. y Bermúdez, J. (2000). Escala de autoeficacia general: datos psicométricos de la adaptación para población española. Psicothema, 12(Suppl. 2), 509-513.

Pollet, M., Egido, A., Le Moine-Perret, A. y Gueben, L. (2016). Personalidad resistente y estrés en los bomberos: vínculos entre las dificultades psicosociales presentes en la actividad y la salud psicológica. Revista de Psicología, 12(23), 25-37. https://repositorio.uca.edu.ar/ handle/123456789/6138 
Ponce-Carbajal, N., Tristán, J. L., Jaenes, J. C., Rodenas, L. T. y Peñaloza, R. (2020). El impacto del contexto social sobre la personalidad resistente en deportistas universitarios de México. Cuadernos de Psicología del Deporte, 20(2), 201-217.

Remor, E., Amorós, M., y Carrobles, J. A. (2006). El optimismo y la experiencia de ira en relación con el malestar físico. Anales de Psicología, 22(1), 37-44.

Ríos, M., Sánchez, J. y Godoy, C. (2010). Personalidad resistente, autoeficacia y estado general de salud en profesionales de Enfermería de cuidados intensivos y urgencias. Psicothema, 22(4), 600-605.

Scheier, M. F., Carver, C. y Bridges M. W. (1994). Distinguishing optimism from neuroticism (and trait anxiety, self-mastery, and self-esteem): A reevaluation of the Life Orientation Test. Journal of Personality and Social Psychology, 67, 1063-1078.

Selye, H. (1956). The stress of life. McGraw-Hill.

Serrato, L. H. (2017). Propiedades psicométricas del cuestionario construido para evaluar personalidad resistente en deportistas (PER-D). Cuadernos de Psicología del Deporte, 17(3), 25-34.

Sheard, M. y Golby, J. (2010). Personality hardiness differentiates elite-level sport performers. International Journal of Sport and Exercise Psychology, 8(2), 160-169. 10.1080/1612 197X.2010.9671940

Shifren, K., Bauserman, R., Blackwood, J., Coles, A. y Hillman, A. (2015). Personality and Mental Health: A Comparison of Emerging Adult Women from Divorce and Intact Families. Journal of Adult Development, 22(4), 221-229. 10.1007/s10804-015-9213-6

Silva-Junior, R. F., Alves, E. C., Santos, K. O., Santos, S. P., Barbosa, H. A., Siqueira, L. G., Torres, J. P. R. y Silva, C. S. (2020). Hardy personality and associated factors in health professionals active in services that treat critical patients. Ciência \& Saúde Coletiva, 25(1), 199-209. 10.1590/1413-81232020251.29442019

Sinclair, R. R. y Tetrick, L. E. (2000). Implications of item wording for hardiness structure, relation with neuroticism, and stress buffering. Journal of Research in Personality, 34(1), 1-25. 10.1006/ jrpe.1999.2265

Sociedad Mexicana de Psicología. (2007). Código ético del psicólogo. (4ª . edición). Trillas.

Vinaccia, S., Contreras, F., Palacio, C., Marín, A., Tobon, S. y Moreno-San Pedro, E. (2005). Disposiciones fortalecedoras: personalidad resistente y autoeficacia en pacientes con diagnóstico de artritis reumatoide. Terapia Psicológica, 23(1), 15-24. 
Vollrath, M. E. (2006). Handbook of personality and health. Wiley and Sons.

Wang, J. y Wang, X. (2012). Structural Equation Modeling: Applications Using Mplus. Wiley Online Library. 10.1002/9781118356258

Wang, X., Wong, J. Y., Zhai, L., Wu, R., Huang, T., He, R., Xiao, Y., Yu, Y., Kong, X., Zhou, X. y Yang, H. (2019). To approach or to avoid? Motivation differentially mediates the effect of hardiness on depressive symptoms in Chinese military personnel. BioMed Research International, 2019, 1-7. 10.1155/2019/7589275

Weigold, I. K., Weigold, A., Kim, S., Drakeford, N. M. y Dykema, S. A. (2016). Assessment of the psychometric properties of the Revised Academic Hardiness Scale in college student samples. Psychological Assessment, 28(10), 1207-1219. 10.1037/pas0000255

Williams, P. G., Smith, T. W., Gunn, H. E. y Uchino, B. N. (2011). Personality and stress: Individual differences in exposure, reactivity, recovery, and restoration. En R. Contrada, y A. Baum (eds.), Handbook of stress science (pp. 231-246). Springer.

Zhang, L. (2011). Hardiness and the big five personality traits among Chinese university students. Learning and Individual Differences, 21(1), 109-113. 10.1016/j.lindif.2010.05.006 


\section{Anexo 1}

\section{Cuestionario de personalidad resistente adaptación mexicana}

\section{Instrucciones:}

Indique el nivel de acuerdo o en desacuerdo que usted tiene en las siguientes situaciones;

\section{Formas de respuesta}

$1=$ Totalmente en desacuerdo

$2=$ En desacuerdo

3= De acuerdo

4= Totalmente de acuerdo

\begin{tabular}{|c|c|c|c|c|}
\hline & 1 & 2 & 3 & 4 \\
\hline \multicolumn{5}{|l|}{$\begin{array}{l}\text { 1. Me involucro seriamente en lo que hago, pues es la mejor manera para } \\
\text { alcanzar mis propias metas. }\end{array}$} \\
\hline \multicolumn{5}{|l|}{$\begin{array}{l}\text { 2. Elijo los trabajos que suponen para mí una nueva experiencia, aunque } \\
\text { requiera mayor esfuerzo. }\end{array}$} \\
\hline \multicolumn{5}{|l|}{ 3. Hago todo lo que puedo para asegurarme de tener el control. } \\
\hline \multicolumn{5}{|l|}{$\begin{array}{l}\text { 4. Considero que el trabajo que realizo es de valor para la sociedad y no me } \\
\text { importa dedicarle todos mis esfuerzos. }\end{array}$} \\
\hline \multicolumn{5}{|l|}{ 5. En mi vida me atraen preferentemente las nuevas experiencias. } \\
\hline \multicolumn{5}{|l|}{ 6. Las cosas solo se consiguen a base de esfuerzo personal. } \\
\hline \multicolumn{5}{|l|}{ 7. Realmente me preocupo y me identifico con mi trabajo. } \\
\hline \multicolumn{5}{|l|}{$\begin{array}{l}\text { 8. En mi trabajo me atraen aquellas tareas y situaciones que implican un } \\
\text { desafío personal. }\end{array}$} \\
\hline \multicolumn{5}{|l|}{ 9. El control de las situaciones es lo único que garantiza el éxito. } \\
\hline \multicolumn{5}{|l|}{ 10. Mi vida cotidiana me satisface y hace que me dedique totalmente a ella. } \\
\hline \multicolumn{5}{|l|}{ 11. En la medida que puedo trato de tener nuevas experiencias en mi vida. } \\
\hline \multicolumn{5}{|l|}{ 12. Las cosas salen bien cuando las preparas a conciencia. } \\
\hline \multicolumn{5}{|l|}{ 13. Dentro de lo posible busco situaciones nuevas y diferentes en mi vida. } \\
\hline \multicolumn{5}{|l|}{ 14. Mis propias ilusiones son las que hacen que siga adelante con mi vida. } \\
\hline 15. Cuando se trabaja seriamente y a fondo se controlan los resultados. & & & & \\
\hline
\end{tabular}

